\title{
A Serine Protease Homolog Negatively Regulates TEP1 Consumption in Systemic Infections of the Malaria Vector Anopheles gambiae
}

\author{
Hassan Yassine $^{a} \quad$ Layla Kamareddine $^{a}$ Soulaima Chamat $^{b}$ \\ George K. Christophides ${ }^{c}$ Mike A. Osta ${ }^{a}$ \\ ${ }^{a}$ Department of Biology, American University of Beirut, Beirut, and ${ }^{b}$ Laboratory of Immunology, Faculty of Public \\ Health, Lebanese University, Fanar, Lebanon; ' Department of Life Sciences, Imperial College London, London, UK
}

\section{Key Words}

Anopheles gambiae - Clip domain serine proteases .

Mosquito innate immunity . Complement-like protein .

Systemic infections

\begin{abstract}
Clip domain serine protease homologs are widely distributed in insect genomes and play important roles in regulating insect immune responses, yet their exact functions remain poorly understood. Here, we show that CLIPA2, a clip domain serine protease homolog of Anopheles gambiae, regulates the consumption of the mosquito complement-like protein TEP1 during systemic bacterial infections. We provide evidence that CLIPA2 localizes to microbial surfaces in a TEP1-dependent manner whereby it negatively regulates the activity of a putative TEP1 convertase, which converts the full-length TEP1-F form into active TEP1 $1_{\text {cut }}$. CLIPA2 silencing triggers an exacerbated TEP1-mediated response that significantly enhances mosquito resistance to infections with a broad class of microorganisms including Plasmodium berghei, Escherichia coli and the entomopathogenic fungus Beauveria bassiana. We also provide further evidence for the existence of a functional link between TEP1 and activation of hemolymph prophenoloxidase during systemic infections.
\end{abstract}

Interestingly, the enhanced TEP1-mediated immune response in CLIPA2 knockdown mosquitoes correlated with a significant reduction in fecundity, corroborating the existence of a trade-off between immunity and reproduction. In sum, CLIPA2 is an integral regulatory component of the mosquito complement-like pathway which functions to prevent an overwhelming response by the host in response to systemic infections.

(c) 2014 S. Karger AG, Basel

\section{Introduction}

Clip domain serine proteases (CLIPs) are central components of extracellular enzymatic cascades in arthropods that regulate several immune responses including antimicrobial peptide expression through the Toll pathway [1], coagulation [2] and melanization [3]. CLIPs contain a C-terminal chemotrypsin-like serine proteinase domain and 1 or more $\mathrm{N}$-terminal clip domains unique to arthropods. Some CLIPs, also known as serine protease homologs (SPHs), have substitutions of 1 or more residues of their catalytic triad rendering them noncatalytic [4]. Despite lacking enzymatic activity, SPHs seem to play important regulatory roles in mosquito immune respons-

\section{KARGER}

E-Mail karger@karger.com

www.karger.com/jin
(C) 2014 S. Karger AG, Basel

1662-811X/14/0066-0806\$39.50/0
Dr. Mike A. Osta

Department of Biology

American University of Beirut

Bliss Street, Beirut 11072020 (Lebanon)

E-Mail mo07@aub.edu.lb 
es. In melanization, efficient cleavage and activation of prophenoloxidase (PPO) by $\mathrm{PPO}$-activating enzymes require SPHs as cofactors [5-7]. SPHs have also been involved in cell adhesion [8] as well as in bacterial opsonization [9].

Insect genomes contain several SPHs with clip domains (clip-SPHs): 14 genes were identified in Drosophi$l a$, whereas 21,11 and 21 genes were identified in the mosquito vectors Anopheles gambiae, Aedes aegypti and Culex quinquefasciatus, respectively $[10,11]$. These genes have undergone extensive diversification between insects as evidenced by the identification of only 3 genes common to the 3 aforementioned mosquito species and 2 genes common to both mosquitoes and Drosophila [10]. Despite the widespread occurrence of clip-SPHs in insect genomes and their implication in a variety of immune responses in different model insects, their modes of action remain poorly understood.

Phylogenetic analysis of mosquito clip-SPH sequences revealed 2 subfamilies termed A and E (for CLIPA and CLIPE) with the former containing the majority of genes [11]. In the malaria vector A. gambiae, SPCLIP1, a member of the CLIPE subfamily, was recently shown to act as a key regulator of the accumulation of the complement C3-like protein TEP1 on surfaces of Plasmodium ookinetes and bacteria leading to microbial lysis or melanization [12]. TEP1 is the hallmark of mosquito immune effector responses to both rodent [13] and human [14, 15] malaria parasites, as well as to systemic infections with bacteria [14, 16, 17] and entomopathogenic fungi [18]. TEP1 exists in the hemolymph as a full-length form called TEP1-F and a proteolytically processed form called TEP $1_{\text {cut }}$ that forms a complex with 2 leucine-rich repeat proteins, LRIM1 and APL1C $[19,20]$. SPCLIP1 seems to function as a positive regulator of a yet unidentified TEP1 convertase that catalyzes TEP1-F cleavage to the active TEP $1_{\text {cut }}$ form [12]. Here, we show that another clip-SPH of subfamily A, termed CLIPA2, acts as a negative regulator of the mosquito complement-like pathway to control the extent of TEP1-F conversion to TEP $1_{\text {cut }}$ during immune responses. We have previously reported that CLIPA2 knockdown $(\mathrm{kd})$ induces the melanization of Plasmodium berghei ookinetes in the basal labyrinth of the midgut epithelium [21]. We provide evidence that this phenotype is due to an enhanced TEP 1 activity in CLIPA2 $\mathrm{kd}$ mosquitoes which renders them more resistant to bacterial and fungal infections as well. Our data highlight yet an additional level of complexity in the regulation of the mosquito complement-like pathway and inform a broader role of clip-SPHs in insect immune responses.

\section{Materials and Methods}

\section{Ethics Statement}

This study was carried out in accordance with the recommendations in the Guide for the Care and Use of Laboratory Animals of the National Institutes of Health, USA. The Institutional Animal Care and Use Committee (IACUC) of the American University of Beirut approved the animal protocol (permit No. 13-07-268). The IACUC functions in compliance with the Public Health Service Policy on the Humane Care and Use of Laboratory Animals (USA), and adopts the Guide for the Care and Use of Laboratory Animals of the National Institutes of Health, USA.

\section{Anopheles gambiae Rearing}

All experiments were performed with the A. gambiae G3 strain. Briefly, A. gambiae mosquitoes were maintained at $27^{\circ} \mathrm{C}$ and $80 \%$ humidity with a 12-hour day-night cycle. Larvae were reared on tropical fish food, and freshly emerged mosquitoes were collected from larval pans using a vacuum collector. Adult mosquitoes were maintained on $10 \%$ sucrose and given mouse blood (mice were anesthetized with ketamine) for egg production.

\section{Synthesis of Double-Stranded RNA for RNA Interference}

Double-stranded RNAs (dsRNA) for LacZ (control), TEP1 and LRIM1 were synthesized as previously described $[13,20]$. The T7tagged primers (T7 tag underlined) T7-CLIPA2-F, TAATACGACTCACTATAGGGATCCTAACAACGGCACA CTGTGTGA, and T7-CLIPA2-R, TAATACGACTCACTATAGGGTCCTGATCGCCATGA TTGGTGGTGCT, were used to PCR amplify a 504-bp amplicon from plasmid pIEX10-CLIPA2 ${ }^{\mathrm{HIS}}$ (see below) containing the full-length coding sequence of CLIPA2. T7-tagged amplicons were purified with GFX PCR DNA and Gel Band purification Kit (GE Healthcare) and used as templates for dsRNA synthesis using the TranscriptAid T7 High Yield Transcription Kit (Thermo Scientific).

\section{Gene Silencing, Mosquito Infections and Survival Assays}

The $P$. berghei strain (PbGFPCON) constitutively expressing green fluorescent protein (GFP) [22] was maintained in 4- to 5 -week-old BALB/c mice from frozen stocks. For parasite infection assays, freshly emerged mosquitoes were maintained on a $10 \%$ sucrose solution containing gentamycin $(15 \mu \mathrm{g} / \mathrm{ml})$, penicillin $(10$ $\mathrm{U} / \mathrm{ml})$ and streptomycin $(10 \mu \mathrm{g} / \mathrm{ml})$ for the first $12 \mathrm{~h}$ after collection from larval pans, and then were kept on $10 \%$ sucrose for the rest of the experiment. This short antibiotic treatment helps eliminate or reduce the impact of microbes contracted by adults from larval cultures, soon after emergence, on parasite development. In vivo gene silencing by RNA interference and parasite counts in dissected mosquito midguts were performed as described previously $[21,23]$.

Mosquito survival following bacterial infections was achieved as previously described [24]. Briefly, dsLacZ-, dsTEP1- and dsCLIPA2treated mosquitoes (not exposed to any antibiotic treatment) were injected with ampicillin-resistant Escherichia coli strain OP-50 [24], suspended in PBS to an optical density at $600 \mathrm{~nm}\left(\mathrm{OD}_{600}\right)=0.4$. A batch of 50 adult female Anopheles were injected per genotype. Mosquito survival rates were scored on a daily basis over 9 days. The Kaplan-Meier survival test was used to calculate the percent survival. Statistical significance of the observed differences was calculated using the log-rank test. Survival assays were repeated at least 3 times. 
Beauveria bassiana spore extractions and natural mosquito infections with $B$. bassiana spores were performed exactly as previously described [18]. Survival test and statistics are as described above. Experiments were repeated at least 3 times using different batches of mosquitoes and fungal spores.

\section{Bacterial Proliferation Assay}

Overnight cultures of $E$. coli OP-50 strain were pelleted by centrifugation, washed 4 times in PBS and resuspended in PBS to an $\mathrm{OD}_{600}=0.4$. Mosquitoes treated with dsLacZ, dsTEP1 and dsCLIPA2 were injected with $69 \mathrm{nl}$ of the prepared bacterial suspension. Two days after injection, 4 batches of 8 mosquitoes each per genotype were ground in $400 \mu \mathrm{l}$ PBS on ice. Serial dilutions of the homogenates were plated onto ampicillin-containing Luria Bertani agar plates and the numbers of colony-forming units (CFUs) scored. The experiment was repeated twice and statistical significance was calculated using the Mann-Whitney test. Means were considered significantly different if $\mathrm{p}<0.05$.

\section{CLIPA2 Cloning, Transfection of Sf9 Cells and Generation of} CLIPA2 Antibody

The entire CLIPA2 open reading frame lacking the endogenous signal peptide was amplified from adult mosquito cDNA using primers CLIPA2-ExpF, GACGACGACAAGATGGAC TACATCC AACAAGAGCAATG, and CLIPA2-ExpR, GAGGAGAAGCCCGGTTTCTCAC GCTGTTTGGAGTGTCCC. The amplified cDNA was sequenced and deposited in GenBank under accession No. KF955283. The underlined sequences are extensions to allow ligase-independent cloning. The amplicon was cloned into $\mathrm{pIEx}^{\mathrm{TM}}-10$ plasmid (Novagen) as a fusion with an $\mathrm{N}$-terminal streptavidin tag and a C-terminal HIS-tag coding for 10 histidines, using a ligase-independent cloning kit (Novagen) according to the manufacturer's protocol. Approximately $1 \times 10^{6}$ exponentially growing Sf9 cells were seeded per well in a 6-well tissue culture plate. Cells were cotransfected with $2 \mu \mathrm{g}$ of plasmid pIEx10CLIPA2 and $0.2 \mu \mathrm{g}$ of pIE1-Neo plasmid (Novagen) using Cellfectin II transfection reagent (Invitrogen), according to the manufacturer's protocol. After $24 \mathrm{~h}$, cells were scraped gently with a syringe plunger, diluted in complete medium [Sf-900 II serum-free medium (Gibco) supplemented with $10 \%$ fetal bovine serum, $50 \mathrm{U} / \mu \mathrm{l}$ penicillin and $50 \mu \mathrm{g} / \mu \mathrm{l}$ streptomycin], and seeded in a 6-well plate at densities of $2 \times 10^{5}, 1 \times 10^{5}, 2.5 \times 10^{4}$ and $1.25 \times 10^{4} \mathrm{cells} / \mathrm{ml}$ in a total volume of $4 \mathrm{ml} /$ well. Cells stably secreting CLIPA2 ${ }^{\mathrm{HIS}}$ were selected with $1 \mathrm{mg} / \mathrm{ml} \mathrm{G} 418$. Clones of resistant cells were analyzed by Western blot for the presence of CLIPA $2^{\mathrm{HIS}}$ in their conditioned medium, and the line with the highest expression was used for protein purification.

Recombinant CLIPA2 ${ }^{\mathrm{HIS}}$ was purified from $80 \mathrm{ml}$ of conditioned media using Talon resin (Clonetech). Talon beads (400 $\mu \mathrm{l}$ ) were washed 3 times in PBS, resuspended in PBS to form a $1: 1$ slurry and then incubated with the conditioned media (supplemented with $0.05 \%$ Triton X-100) on a rotating wheel for $3 \mathrm{~h}$ at room temperature. After washing 4 times with PBS containing $10 \mathrm{mM}$ imidazole, bound proteins were eluted in $4 \mathrm{ml}$ PBS containing $300 \mathrm{mM}$ imidazole, $\mathrm{pH}$ 8.0. Purified recombinant CLIPA $2{ }^{\text {HIS }}$ was used to immunize mice using the TiterMax Gold Adjuvant (Sigma), according to the manufacturer's protocol. CLIPA2 polyclonal serum was purified using the Melon Gel IgG Spin Purification Kit (Thermo Scientific) as described by the manufacturer.

\section{Bioparticle Challenge and Western Blot Analysis}

Adult female mosquitoes were injected each with $69 \mathrm{nl}$ (approx. $4 \times 10^{5}$ bacteria) of a $20 \mathrm{mg} / \mathrm{ml}$ suspension of pHrodolabeled E. coli bioparticles (Invitrogen) in sterile PBS, and hemolymph was collected directly into nonreducing SDS-PAGE sample buffer from groups of 30 mosquitoes at 1,3 and $12 \mathrm{~h}$ after bioparticle injection. Hemolymph proteins were separated by SDS-PAGE and transferred to Immunoblot polyvinylidene fluoride membrane (BioRad) using wet transfer (BioRad). Blots were incubated with mouse anti-CLIPA2, rabbit anti-TEP1 [25] and rabbit anti-PPO6 [26] polyclonal antibodies at dilutions of $1 / 1,000,1 / 1,000$ and 1/2,000, respectively. Anti-mouse and antirabbit IgG horseradish peroxidase-conjugated secondary antibodies (Promega) were used at $1 / 6,000$ and $1 / 12,000$, respectively.

A bioparticle surface extraction assay was performed exactly as previously described [12]. Proteins in the soluble and bound fractions were resolved by nonreducing SDS-PAGE, and Western blot was performed as described above.

To study the effects of TEP1 and LRIM1 kd on CLIPA2 protein levels in the absence of infection, hemolymph was extracted from naive mosquitoes $48 \mathrm{~h}$ after dsRNA injection. Hemolymph proteins were resolved by nonreducing SDS-PAGE, and Western blot was performed as described above. Rabbit anti-LRIM1 polyclonal antibody was used at 1/2,000 [20].

\section{Immunohistochemistry and Fluorescence Microscopy}

Immunolocalization of TEP1 to ookinetes was performed $26 \mathrm{~h}$ after feeding mosquitoes on $P$. berghei-infected mice. Wholemount immunohistochemistry of midguts was performed as previously described [12]. Affinity-purified rabbit anti-TEP1 polyclonal antibody and Alexa-546-conjugated anti-rabbit secondary antibody (Molecular Probes) were used at dilutions of 1/350 and $1 / 800$, respectively. Images were acquired on a Zeiss LSM 710 META confocal microscope.

\section{Bacterial Melanization and Phenoloxidase Assay}

Adult female mosquitoes treated with dsLacZ and dsCLIPA2 were injected with $E$. coli strain OP-50 suspended in PBS to an $\mathrm{OD}_{600}=0.4$. Abdomens were dissected $48 \mathrm{~h}$ after infection, fixed with $4 \%$ paraformaldehyde for $50 \mathrm{~min}$, washed 3 times with $1 \times$ PBS containing $0.05 \%$ Triton X-100 and mounted in Vectashield mounting medium (Vector Laboratories). Melanized bacteria in dissected abdomens (or bacterial clusters) were easily detectable under a light microscope as black bodies of various sizes mainly at the level of the periosteal regions. Abdomens were grouped into 4 empirical categories depending on the intensity of melanization: no detectable melanization; weak melanization (restricted to the periosteal regions); moderate melanization (restricted to the periosteal regions but more pronounced in intensity and size); strong melanization (disseminated throughout the abdomen). Data shown is from 60 abdomens per genotype pooled from 2 independent experiments showing similar distributions. Distributions were compared using the KolmogorovSmirnov test.

The phenoloxidase enzymatic assay was performed $3 \mathrm{~h}$ after mosquito injection with $E$. coli strain OP-50 $\left(\mathrm{OD}_{600}=0.8\right)$ using approximately $5 \mu \mathrm{g}$ of mosquito hemolymph per reaction as described previously [27]. The absorbance at $492 \mathrm{~nm}$ was measured 40 min after incubation with L-3,4-dihydroxyphenylala- 


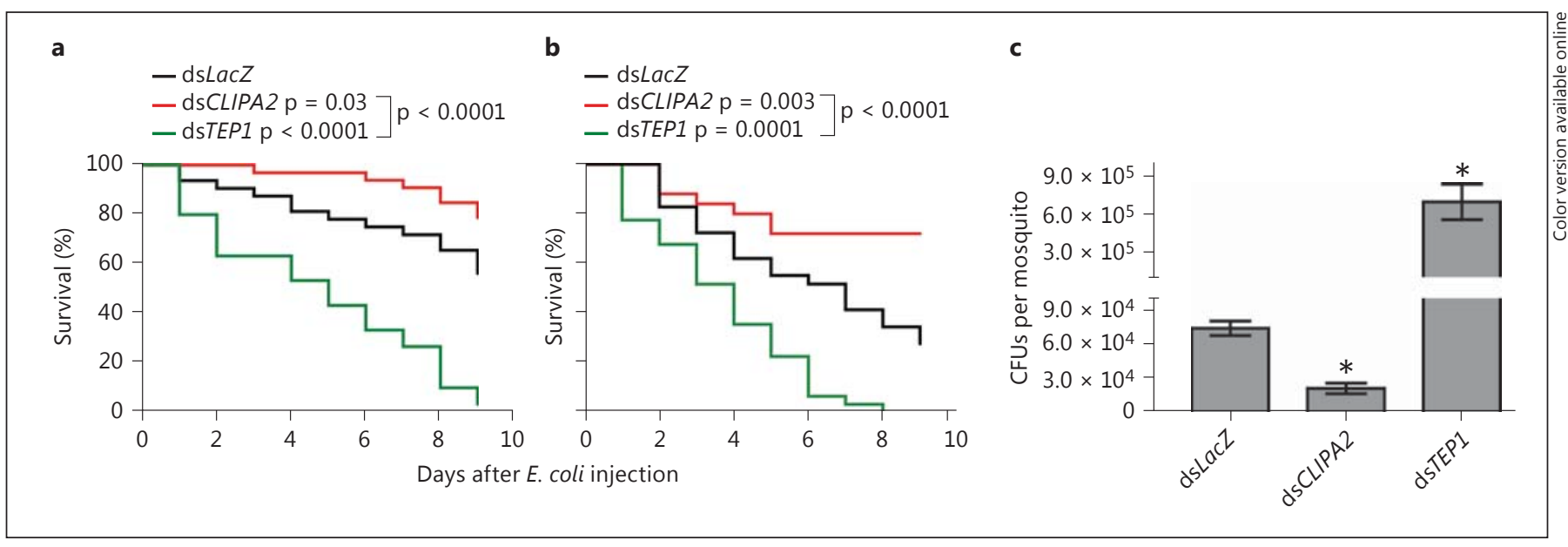

Fig. 1. CLIPA2 $\mathrm{kd}$ mosquitoes are more tolerant and resistant to $E$. coli injections. A. gambiae adult female mosquitoes were injected with $E$. coli strain OP-50 $\left(\mathrm{OD}_{600}=0.4\right) 4$ days after silencing with dsLacZ (control), dsTEP1 and dsCLIPA2. a, b Dead mosquitoes were counted daily over a period of 9 days after bacterial challenge. Percent survival was calculated using the Kaplan-Meier method for 2 independent representative experiments. Statistical significance was calculated using the log-rank test. Survival curves were considered to be significantly different if $\mathrm{p}<0.05$. $\mathrm{c}$ Two days after injection of $E$. coli $\left(\mathrm{OD}_{600}=0.4\right), 5$ batches of 8 mosquitoes each per indicated genotype were ground in PBS, and live bacterial counts were determined by plating serial dilutions on LB plates supplemented with ampicillin. Graphs represent mean CFU values per mosquito with their standard errors. Statistical significance was calculated using the Mann-Whitney test, and asterisks indicate $\mathrm{p}<0.05$. Data shown are from 2 independent biological experiments. nine (Sigma) in a Multiskan Ex microplate reader (ThermoLabsystems). The experiment was repeated 3 times using different batches of mosquitoes and E. coli cultures.

\section{Mosquito Fecundity Assay}

dsLacZ and dsCLIPA2 female mosquitoes were injected each with $69 \mathrm{nl}$ of an E. coli suspension in $\mathrm{PBS}$ at $\mathrm{OD}_{600}=0.4$. Noninjected and PBS (sterile)-injected wild-type naive mosquitoes were used as controls. Mosquitoes from the different experimental groups were allowed to feed on the same naive mouse 4 days after injection. Blood-fed females were placed individually in paper cups, and the eggs laid were counted using a stereoscope. Eggs from a total of at least 72 individual mosquitoes were counted per experimental group from 2 independent experiments. In each experiment, all mosquitoes used were from the same batch and of the same age to reduce the number of variables. Statistical analysis was performed using the Mann-Whitney test. Medians were considered significant if $\mathrm{p}<0.05$.

\section{Results}

\section{CLIPA2 kd Mosquitoes Are More Tolerant and Resistant to Systemic Microbial Infections}

It was previously proposed that CLIPA2 acts as a negative regulator of A. gambiae melanization response following the observation that CLIPA2 silencing triggered partial melanization of $P$. berghei ookinetes in the basal labyrinth of the mosquito midgut epithelium [21]. To gain further insight into CLIPA2 function and its relation to melanization, we monitored the effect of silencing this gene on mosquito susceptibility to systemic bacterial infections with E. coli strain OP-50. Using this same strain as a model pathogen, it was suggested that melanization is not required for $A$. gambiae defense against systemic bacterial infections [27]. Hence, if CLIPA2 functions strictly as a negative regulator of melanization, as previously proposed [21], then CLIPA2 $\mathrm{kd}$ mosquitoes are expected to show a similar susceptibility to $E$. coli as $L a c Z$ $\mathrm{kd}$ controls. To address this point, LacZ, CLIPA2 and TEP1 kd mosquitoes were injected with live E. coli $\left(\mathrm{OD}_{600}=0.4\right)$, and their survival was scored over a 9-day period. TEP1 kd mosquitoes served as positive controls since they are susceptible to $E$. coli injections $[14,17]$. Interestingly, CLIPA2 kd mosquitoes were consistently more tolerant to E. coli compared to controls, whereas TEP1 kd mosquitoes succumbed quickly to the infection as expected (fig. 1a, b). We then measured the resistance of these mosquito genotypes to the injected $E$. coli strain by scoring the bacterial CFUs in mosquito lysates 2 days after injection. Our results revealed that CLIPA2 kd mosquitoes harbored significantly fewer CFUs than LacZ kd 
controls; mean values were 21,000 and 74,000 CFUs per mosquito, respectively (fig. 1c). In contrast, TEP1 kd resulted in strong bacterial proliferation with a mean value of 694,000 CFUs per mosquito. Hence, according to our previous argument, these results do not support a strict role for CLIPA2 in the regulation of melanization because the susceptibility profile of CLIPA2 kd mosquitoes to $E$. coli deviated significantly from that of $L a c Z \mathrm{kd}$ controls, whereas melanization was previously shown not to affect mosquito tolerance or resistance to the same E. coli strain [27]. CLIPA2 kd mosquitoes also exhibited enhanced tolerance to natural infections with the entomopathogenic fungus $B$. bassiana compared to controls (online suppl. fig. 1; see www.karger.com/doi/10.1159/000363296 for all online suppl. material). In sum, our results suggest that CLIPA2 is likely acting as a negative regulator of a key immune effector response with broad antimicrobial activity, other than melanization.

\section{CLIPA2 Controls TEP1 Consumption during Systemic} Infections

Based on the phenotype exhibited by CLIPA2 kd mosquitoes, we hypothesized that CLIPA2 may act as a negative regulator of the complement-like pathway during immune responses for the following reasons: first, TEP1 is, so far, the only effector protein shown to be required for defense against a broad class of microorganisms including Plasmodium parasites [13-15], bacteria [14, 16, 17] and fungi [18]; second, TEP1 kd almost abolished hemolymph PPO activation in response to systemic infections with bacteria [12] and B. bassiana [18], suggesting a central role for TEP1 in triggering melanization; third, SPCLIP1, a clip-SPH similar to CLIPA2, acts as a key regulator of the complement-like pathway leading to TEP1 accumulation on microbial surfaces [12]. To assess the role of CLIPA2 in regulating mosquito complement we monitored temporally and quantitatively TEP1 protein levels in the hemolymph following mosquito injections with $E$. coli bioparticles. Hemolymph was extracted from $L a c Z$ and CLIPA2 $\mathrm{kd}$ mosquitoes at 1, 3 and $12 \mathrm{~h}$ after injection of $E$. coli bioparticles and subjected to Western blot analysis using TEP1-specific antibodies. In LacZ kd mosquitoes, TEP1-F was almost depleted from the hemolymph $1 \mathrm{~h}$ after injection but returned to basal levels at later time points (fig. 2a), whereas after silencing CLIPA2 TEP1-F levels were markedly reduced up till $3 \mathrm{~h}$ after injection and remained below the basal level even at the 12hour time point. These results indicate that CLIPA2 silencing triggers a more robust and prolonged consumption ofTEP1-F in response to systemic bacterial infections.
This observed reduction in TEP1-F hemolymph protein is not due to a reduction in TEP1 gene transcript levels because CLIPA2 kd mosquitoes exhibited a similar increase in TEP1 transcripts following bacterial challenge as LacZ kd controls (data not shown).

In a recent study, SPCLIP1 was shown to positively regulate a complement convertase-like activity (termed TEP1 convertase) responsible for the proteolytic conversion of TEP1-F to TEP $1_{\text {cut }}$ [12]. It was clearly demonstrated that SPCLIP1 silencing abolished infection-induced TEP1-F depletion from the hemolymph [28]. TEP1-F levels in bioparticle-injected CLIPA2 kd mosquitoes followed completely opposite dynamics with respect to those reported in SPCLIP1 kd mosquitoes [12], suggesting a role for CLIPA2 in the negative regulation of TEP1 convertase activity. In that case, CLIPA $2 \mathrm{kd}$ would be expected to lead to increased TEP $1_{\text {cut }}$ levels in parallel with the reduction in those of TEP1-F over the examined time range. However, we did not observe such a pattern, but rather TEP $1_{\text {cut }}$ was reduced concomitantly with TEP1-F (fig. 2a). This profile was not surprising to us for the following reasons: first, TEP $1_{\text {cut }}$ is the active form of TEP1 $[19,20]$; second, TEP $1_{\text {cut }}$ localization to microbial surfaces drives several effector responses including opsonization [16, 17], lysis [13] and melanization [12, 18]. Based on these facts, it is unlikely to detect an increase in levels of TEP $1_{\text {cut }}$ in bioparticle-challenged CLIPA2 $\mathrm{kd}$ mosquitoes because it is expected to be consumed in reactions leading to opsonization and melanization of bioparticles.

Interestingly, when the same membrane was reprobed with CLIPA2 antibody, CLIPA2 protein was almost depleted from the hemolymph of LacZ kd control mosquitoes $1 \mathrm{~h}$ after bacterial injection but was replenished at later time points (fig. 2a), indicating a strong consumption of CLIPA2 during immune reactions concomitant with that of TEP1. As expected, CLIPA2 was depleted from the hemolymph of CLIPA2 kd mosquitoes at all time points after injection showing that CLIPA2 antibody is specific. CLIPA2 migrates at approximately $100 \mathrm{kDa}$ which is higher than its predicted $66.75 \mathrm{kDa}$ molecular weight (fig. 2a). It should be noted that all protein samples were analyzed under nonreducing conditions, because, for an unknown reason, the antibody failed to recognize CLIPA2 when samples were treated with reducing agents. Since CLIPs have several disulfide bonds, the absence of a reducing agent might have caused this aberrant migration. Alternatively, the presence of a long stretch of threonine residues in CLIPA2 that constitute putative glycosylation sites (online suppl. fig. 2) may explain this migra- 

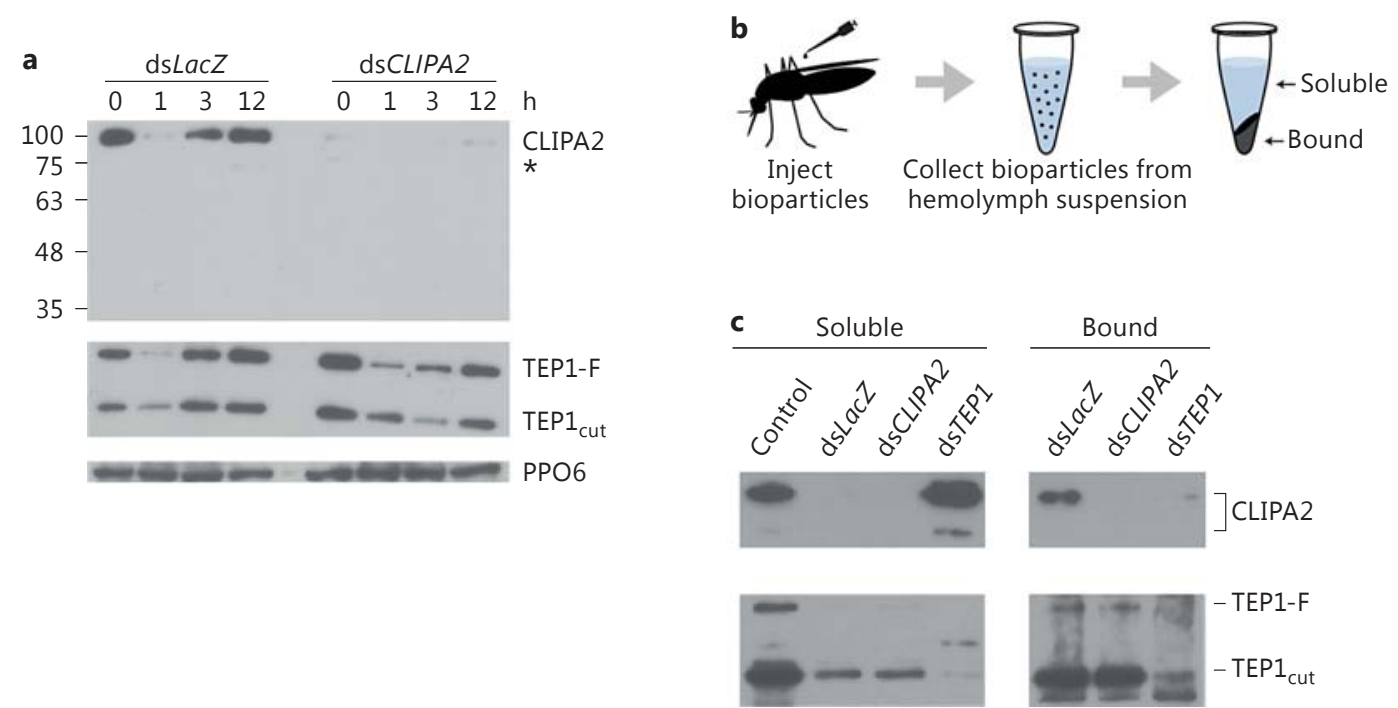

Fig. 2. CLIPA2 negatively regulates TEP1 consumption in immune reactions. a Western blot analysis of hemolymph extracted from dsLacZ and dsCLIPA2 mosquitoes at the indicated time points after injection of $E$. coli bioparticles. TEP1-F and TEP $1_{\text {cut }}$ correspond to the full-length and processed forms of TEP1, respectively. PPO6 was used as a loading control. The asterisk represents a minor CLIPA2 band. b Schematic overview of the bioparticle surface extraction assay. Hemolymph containing E. coli bioparticles was extracted from the indicated mosquito genotypes $20 \mathrm{~min}$ after bioparticle inoculation into the hemocele. Bioparticles were pelleted by centrifugation and the soluble fractions collected. Bacterial pellets were washed with buffer before bound proteins were extracted. c Western blot analysis of the soluble and bound fractions using antibodies against CLIPA2 and TEP1. Hemolymph extracted from the same number of uninjected mosquitoes (control lane) served as a reference for comparing the soluble fractions. The image is representative of 3 independent experiments.

levels from the hemolymph of $L a c Z \mathrm{kd}$ mosquitoes $1 \mathrm{~h}$ after bioparticle injection (fig. 2a) suggests that CLIPA2 is likely to be sequestered on bacterial surfaces together with TEP1. We addressed this point using the bioparticle surface extraction assay that allows the quantitative assessment of interactions occurring between mosquito hemolymph proteins and bacterial surfaces, as previously described [12]. Briefly, E. coli bioparticles were injected into female mosquitoes, and hemolymph was extracted $15 \mathrm{~min}$ after injection. Bioparticles were separated from the hemolymph by centrifugation, washed extensively and their surface-bound proteins eluted for Western blot analysis (fig. 2b). In LacZ kd controls, CLIPA2 was almost exclusively detected in the bound fraction with a barely detectable band appearing in the soluble one (fig. 2c). Interestingly, in TEP1 kd mosquitoes CLIPA2 distribution between both fractions was completely reversed; CLIPA2 was almost absent from the bound fraction and enriched in the soluble fraction, indicating that TEP1 is required for CLIPA2 recruitment to bioparticle surfaces. 
In LacZ kd controls, both TEP $1_{\text {cut }}$ and TEP1-F were more enriched in the bound fraction, with TEP $1_{\text {cut }}$ being by far more abundant (fig. 2c). These results conform with previous observations showing that TEP $1_{\text {cut }}$ is the main form associated with bacterial surfaces $[12,16]$. In CLIPA2 $\mathrm{kd}$ mosquitoes, TEP $1_{\text {cut }}$ and TEP1-F levels were similar to those in $L a c Z \mathrm{kd}$ controls in both the soluble and bound fractions clearly indicating that TEP1 acts upstream of CLIPA2. Based on the data in figure $2 \mathrm{a}, \mathrm{TEP} 1_{\text {cut }}$ was expected to be more abundant in the bound fraction of CLIPA2 $\mathrm{kd}$ mosquitoes relative to LacZ kd controls, but this was not the case. A plausible explanation is that excess TEP $1_{\text {cut }}$ accumulating on bioparticle surfaces in the former genotype triggered their rapid elimination through opsonisation and melanization. These immune reactions render bioparticles nonextractable due to their uptake by hemocytes in the abdomen [32] or adherence to tissues. In support of this argument, we consistently obtained smaller bioparticle pellets after the centrifugation of hemolymph extracts from CLIPA2 kd mosquitoes relative to those from the same number of $L a c Z \mathrm{kd}$ controls (data not shown), indicating a quicker elimination of microbes in the former genotype.

\section{CLIPA2 kd Enhances Hemolymph Phenoloxidase}

Activity in Response to Systemic Bacterial Infections

Bacterial injections into the hemocele of wild-type A. gambiae mosquitoes triggered PPO activation leading to melanization [24, 27]. TEP1 controls tightly the activation of hemolymph PPO, since TEP1 silencing almost abolished PPO activation following bacterial [12] and fungal [18] systemic infections. Furthermore, TEP1 depletion inhibited the infection-induced cleavage of CLIPA8, an essential factor for PPO activation [12]. Consequently, if CLIPA2 is acting indeed as a negative regulator of TEP1 consumption, then silencing its gene is expected to trigger an exacerbated melanotic response to systemic bacterial infections. To address this point, we first scored the intensity of melanization in abdomens of $L a c Z$ and CLIPA2 $\mathrm{kd}$ mosquitoes dissected $48 \mathrm{~h}$ after injection of live E. coli, and grouped abdomens into $4 \mathrm{em}$ pirical categories depending on the size and spatial distribution of melanotic foci. In LacZ kd mosquitoes, the majority of dissected abdomens showed small melanotic foci restricted to the periosteal regions (fig. 3a, c). Periosteal hemocytes were shown to aggregate in these regions during systemic infections in order to sequester microbes present in the hemolymph [32]. However, the majority of abdomens from CLIPA2 kd mosquitoes showed larger melanotic foci with a more scattered distribution (fig. 3b, c) indicating an exacerbated melanotic response. To further support these observations, phenoloxidase activity was measured in LacZ, CLIPA2 and TEP1 kd mosquitoes $3 \mathrm{~h}$ after injection of live E. coli $\left(\mathrm{OD}_{600}=0.8\right)$. CLIPA2 $\mathrm{kd}$ mosquitoes exhibited a significant 2.4-fold increase in phenoloxidase activity compared to LacZ controls (fig. 3d), which explains the intense bacterial melanization observed in their abdomens. TEP1 kd almost abolished PPO activation as previously reported [12]. In sum, these results strongly suggest that enhanced TEP1 consumption in systemic infections of CLIPA2 kd mosquitoes is responsible for the abnormal phenoloxidase activity measured in their hemolymph.

\section{CLIPA2 Is a Component of the Mosquito}

Complement-Like Pathway

TEP $1_{\text {cut }}$ is stabilized in the hemolymph by a complex of 2 leucine-rich repeat proteins LRIM1 and APL1C $[19,20]$. Silencing either of these proteins in naive mosquitoes triggered the loss of TEP $1_{\text {cut }}$ from the hemolymph compartment and its accumulation on host tissues. Interestingly, TEP $1_{\text {cut }}$ accumulation on tissues was concomitant with almost the depletion of SPCLIP1 from the hemolymph, indicating that SPCLIP1 is tightly linked to the TEP $1_{\text {cut }}$ function [12]. Thus, we examined whether CLIPA2 steady-state levels in the hemolymph show similar dependency on TEP $1_{\text {cut }}$ under naive conditions. Naive mosquitoes were injected with dsRNAs corresponding to the indicated genes, and hemolymph was extracted $48 \mathrm{~h}$ later and subjected to Western blot analysis. TEP $1 \mathrm{kd}$ showed no effect on CLIPA2 steady-state levels which were similar to those in the control group (fig. 4). However, LRIM1 kd almost abolished CLIPA2 presence in the hemolymph concomitant with the loss of TEP $1_{\text {cut }}$ (fig. 4), suggesting that CLIPA2 is following the latter to the tissues. Cosilencing TEP1 and LRIM1 restored the baseline levels of CLIPA2 suggesting that it functions downstream of TEP $1_{\text {cut }}$ CLIPA2 kd did not affect the steady-state levels of LRIM1 or TEP1. Altogether, these results clearly indicate that, similar to SPCLIP1 [12], CLIPA2 is tightly linked to the TEP $1_{\text {cut }}$ function in both infected and naive conditions, and it constitutes as such an integral component of the TEP1 pathway.

\section{CLIPA2 Silencing Exacerbates TEP1-Mediated Killing} of P. berghei Ookinetes

CLIPA2 silencing was previously shown to trigger partial melanization of $P$. berghei ookinetes in susceptible mosquitoes of strain G3 [21]. Based on the data 
Fig. 3. CLIPA2 $\mathrm{kd}$ enhances the mosquito melanotic response to injected E. coli. Abdomens were dissected from dsLacZ and dsCLIPA2 mosquitoes $48 \mathrm{~h}$ after injection of E. coli strain OP-50 $\left(\mathrm{OD}_{600}=0.4\right)$, and the intensity of melanization (melan.) was scored empirically as described in the Methods section. Bright-field images of dsLacZ (a) and dsCLIPA2 (b) abdomens show weak and strong bacterial melanization phenotypes, respectively. Arrowheads indicate melanized clusters of bacteria. Scale bars are $10 \mu \mathrm{m}$. c Distribution of melanization intensity scored in dissected abdomens pooled from 2 independent biological experiments. Distributions were compared using the Kolmogorov-Smirnov test. d Phenoloxidase (PO) enzymatic activity (detected as absorbance at $492 \mathrm{~nm}$, $\mathrm{OD}_{492}$, after conversion of L-3,4-dihydroxyphenylalanine) was measured in hemolymph extracted from dsCLIPA2 and dsTEP1 mosquitoes and compared to that in dsLacZ controls $3 \mathrm{~h}$ after injection of $E$. $\operatorname{coli}\left(\mathrm{OD}_{600}=0.8\right)$. The graphs show phenoloxidase activity measured $40 \mathrm{~min}$ after addition of L-3,4-dihydroxyphenylalanine. Means were calculated from 3 independent biological experiments. Error bars represent standard deviation of the mean. Statistical analysis was performed using Student's t test, and differences were considered to be significant if $\mathrm{p}<0.05$.
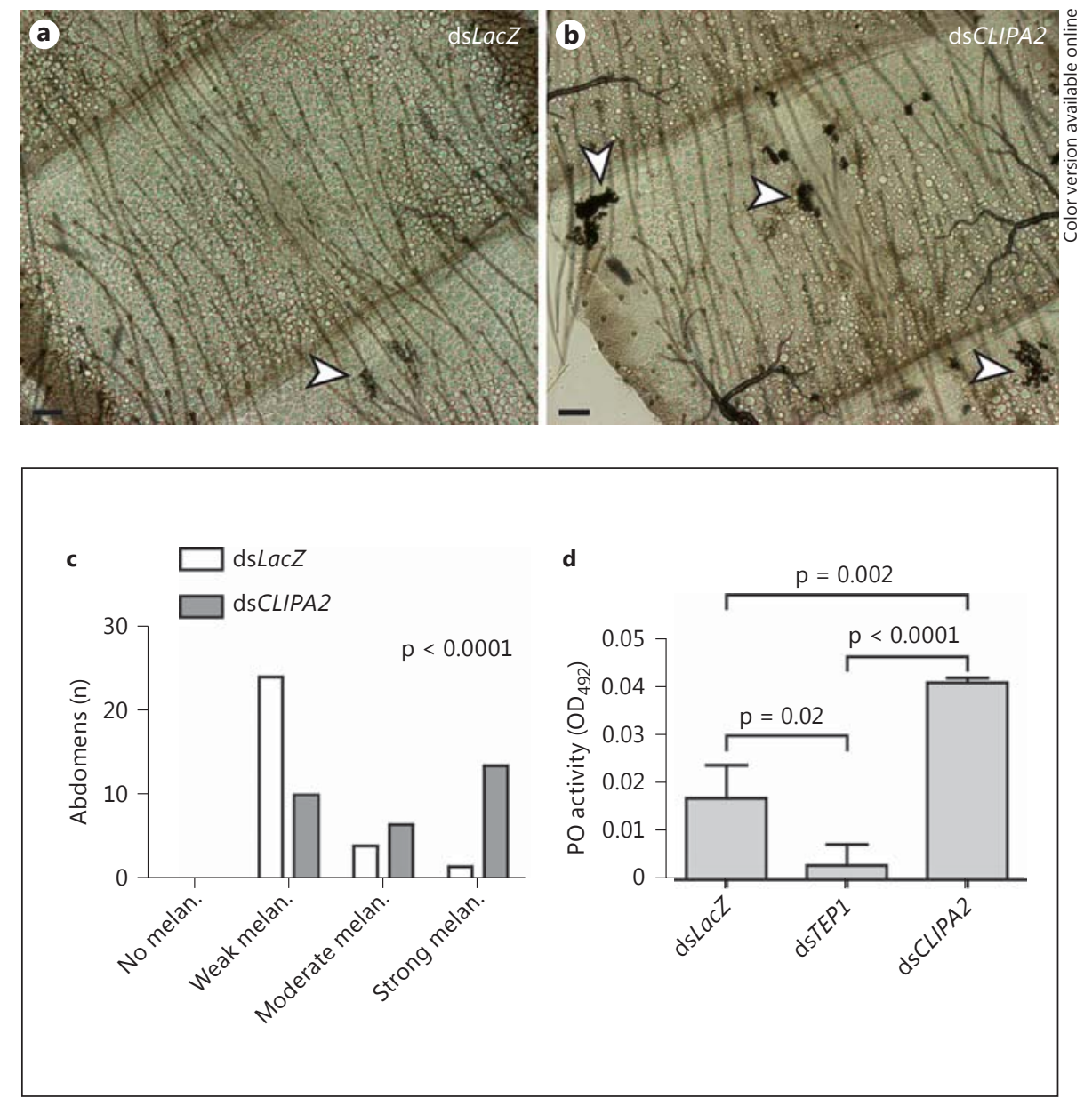

presented so far, a plausible explanation to this phenotype is an exaggerated TEP1-mediated killing of ookinetes leading to melanization. Before addressing this point, we first reassessed the effect of CLIPA2 silencing on ookinete melanization in our G3 mosquitoes for the following reasons: first, the melanotic phenotype previously reported for CLIPA2 silencing was partial ( $8 \%$ melanized ookinetes) [21]; second, the colony has probably passed through several bottlenecks which might have altered the CLIPA2 $\mathrm{kd}$ phenotype. Therefore, LacZ, CLIPA 2 and TEP1 kd mosquitoes were allowed to feed on $P$. berghei-infected mice and their midguts dissected 7 days later to score for melanized ookinetes and live oocysts. CLIPA $2 \mathrm{kd}$ mosquitoes exhibited a significant increase in numbers of melanized ookinetes ( $\mathrm{p}_{\mathrm{Mo}}$ $<0.001)$ concomitantly with a reduction in numbers of live oocysts $\left(\mathrm{p}_{\mathrm{Lo}}<0.001\right.$; fig. $\left.5 \mathrm{a}, \mathrm{b}\right)$ relative to controls. However, the numbers of melanized ookinetes were significantly higher in our current G3 mosquitoes than those reported previously in the same strain (56.2 vs. $8 \%$, respectively), possibly due to genotypic changes at specific loci resulting from colony bottlenecks. TEP1 $\mathrm{kd}$, used as a positive control, triggered a significant increase in numbers of live oocysts as previously reported [13].

To determine whether CLIPA2 regulates the extent of ookinete killing by TEP1, we examined the effect of silencing CLIPA2 on the numbers of TEP1-stained ookinetes. Accordingly, midguts were dissected from $L a c Z$ and CLIPA2 $\mathrm{kd}$ mosquitoes at $26 \mathrm{~h}$ after an infectious blood meal and immunostained with TEP1 antibody. The numbers of ookinetes positive for GFP (GFP+), TEP1 (TEP+), both GFP and TEP1 (GFP+l $\mathrm{TEP}+$ ) or melanin (i.e. melanized) were scored using confocal microscopy (fig. 5c, d). Midguts dissected from CLIPA2 kd mosquitoes showed an overall significant increase in TEP1+ parasites (TEP1+ and GFP+/ TEP1+ and melanized) in comparison to controls, in 2 
independent biological experiments (fig. 5e): 63.9 versus $46.1 \%$ in experiment 1 and 59.8 versus $25.6 \%$ in experiment 2, respectively. Melanized ookinetes were considered TEP1+ since TEP1 is required for ookinete melanization, and PPO localizes only on the surface of TEP1-labeled ookinetes [13]. Hence, these results suggest that the enhanced ookinete killing in CLIPA2 kd mosquitoes, manifested in the form of melanized ookinetes, is due to an exaggerated TEP1-mediated response to these parasite stages.

\section{CLIPA2 kd Reduces Mosquito Fecundity in Response to Systemic Bacterial Infections}

An exacerbated immune response may affect host fitness through the redistribution of energy resources allocated to other vital physiological processes or through reduced tolerance to the microbe driven by immune-mediated tissue damage [33]. CLIPA2 kd mosquitoes trigger an enhanced TEP1-mediated immune response to bacterial, fungal and Plasmodium infections yet they seem to tolerate or endure the infection even better than controls, at least in terms of their survival rate. Since survival is one amongst several fitnessrelated parameters, we looked for other hidden costs in particular reproductive success, especially that previous studies in A. gambiae pointed to the existence of a tradeoff between ookinete killing and oogenesis [34]. To address this point, $L a c Z$ and CLIPA $2 \mathrm{kd}$ mosquitoes were injected with an E. coli suspension $\left(\mathrm{OD}_{600}=0.4\right)$ and allowed to recover during 4 days after the injection process. Wild-type mosquitoes injected with sterile PBS were also given the same recovery period. Noninjected naive mosquitoes were used as controls. Four days after recovery, mosquitoes from the 4 experimental groups were fed on the same naive mouse for an equal amount of time, and eggs laid by individually sorted females were counted using a stereoscope. Injection of sterile PBS reduced significantly mosquito fecundity relative to control, suggesting that wounding per se reduces fecundity (fig. 6). LacZ kd mosquitoes injected with E. coli exhibited further reduction in egg production compared to the PBS-injected group ( $\mathrm{p}<0.0001)$, possibly due to additional cost imposed by infection and immune activation. Interestingly, E. coli-injected CLIPA2 $\mathrm{kd}$ mosquitoes produced even fewer eggs than their LacZ kd controls ( $\mathrm{p}<0.0001)$. Since CLIPA2 silencing renders mosquitoes more resistant to systemic $E$. coli injections (fig. 1c), the lower fecundity of these genotypes reflects most probably the cost of triggering an overwhelming TEP1-mediated systemic response.

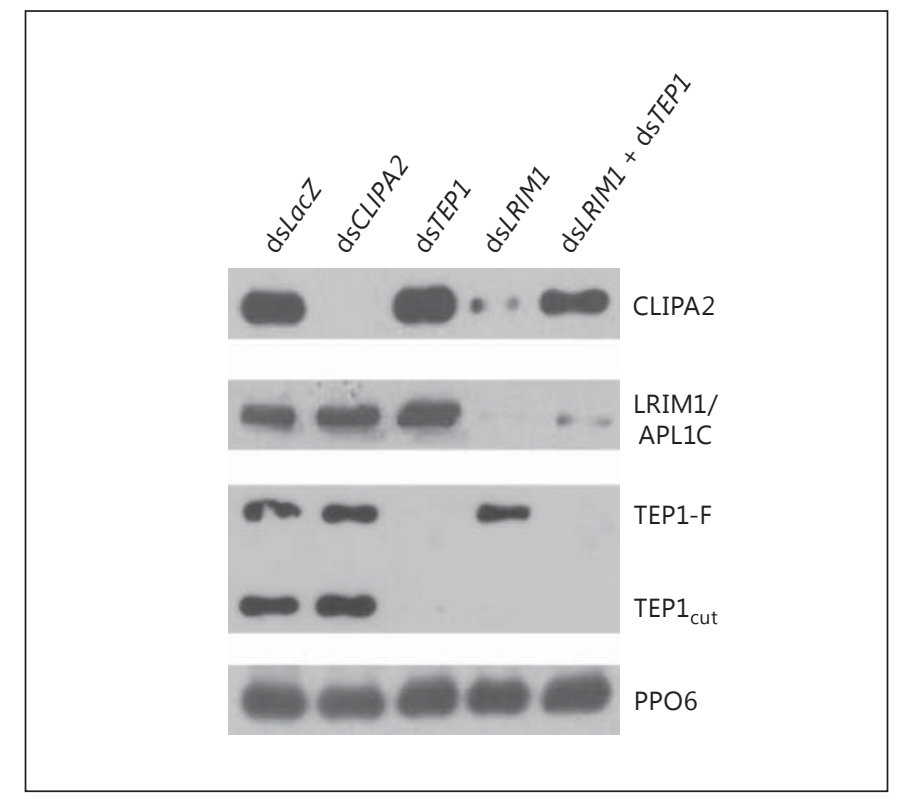

Fig. 4. CLIPA2 is a component of the complement-like pathway. Western blot analysis of hemolymph extracted 2 days after injecting naive mosquitoes with the indicated dsRNAs. The membrane was probed with antibodies against CLIPA2, LRIM1, TEP1 and PPO6 as loading control. The image is representative of 3 independent biological experiments.

\section{Discussion}

Clip-SPHs play important regulatory roles in insect immune responses. Here, using the bacterial bioparticle infection system we demonstrate that CLIPA2 localizes to microbial surfaces whereby it negatively regulates the consumption of mosquito TEP1 during systemic infections, adding a new level of complexity to complement regulation in A. gambiae. CLIPA2 functions to avoid an overwhelming TEP1-mediated response that may be energetically costly for the host. Such a response is indeed taking place in CLIPA2 $\mathrm{kd}$ mosquitoes according to the following observations: abnormally high hemolymph phenoloxidase activity in response to systemic E. coli injections; enhanced tolerance to E. coli and B. bassiana infections, and increased resistance to E. coli and P. berghei ookinetes.

Unfortunately, we were not able to investigate the potential interaction between CLIPA 2 and TEP 1 in vivo by immunoprecipitation assays, since the CLIPA2 antibody in hand does not recognize the native protein. This also hampered efforts to localize CLIPA2 on ookinete surfaces using fluorescent microscopy. However, based on our 


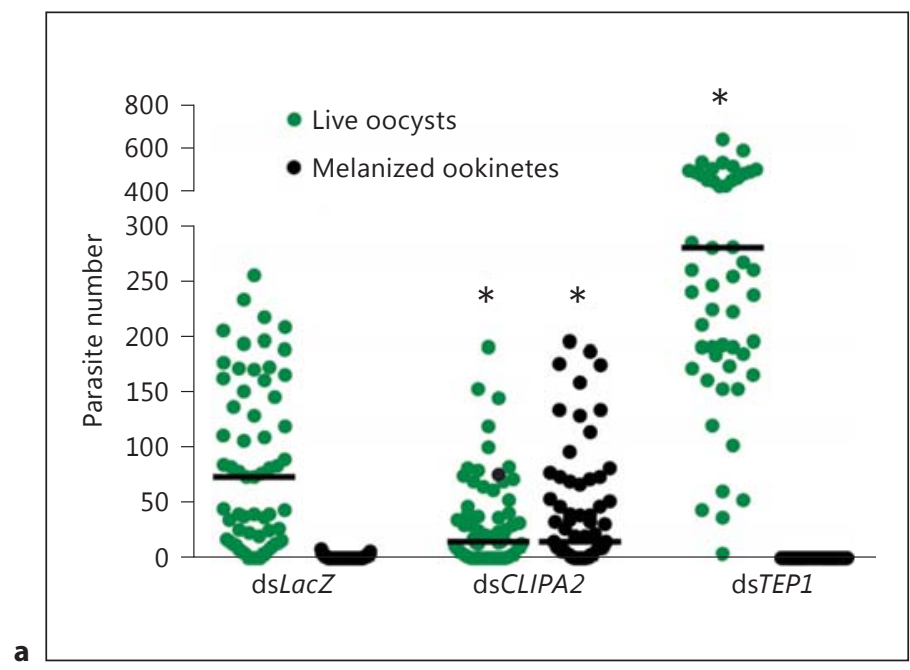

\begin{tabular}{|llrrrrrrr|}
\hline Gene kd & $\mathrm{n}$ & Mo & $\mathrm{p}_{\text {Mo }}$ & Mo, \% & Lo & $\mathrm{p}_{\text {Lo }}$ & \multicolumn{1}{c|}{ Tp } & $\mathrm{p}_{\text {Tp }}$ \\
\hline LacZ & 65 & 0 & & 0.9 & 74 & & 74 & \\
CLIPA2 & 67 & 15 & $<0.001$ & 56.2 & 15 & $<0.001$ & 57 & 0.675 \\
TEP1 & 62 & 0 & & 0 & 283 & $<0.001$ & 283 & $<0.001$ \\
\hline
\end{tabular}

Fig. 5. TEP1-mediated ookinete killing is increased in CLIPA2 kd mosquitoes. a Scatter plot of live GFP-expressing P. berghei oocysts (green circles; colors in the online version only) and dead melanized ookinetes (black circles) scored in the midguts of dsLacZ, dsCLIPA2 and dsTEP1 mosquitoes 7 days after infection. Lines indicate median parasite numbers. The Mann-Whitney test was used to compare dsCLIPA2 and dsTEP1 mosquitoes with $\mathrm{ds} L a c Z$ controls, and asterisks indicate p values less than 0.05 . Data were pooled from 3 independent biological experiments. b Tabulated data of a showing the median numbers of melanized ookinetes (Mo), live oocysts (Lo) and total parasites (Tp) per midgut. $\mathrm{p}$ values were calculated by the Mann-Whitney test. c, d Representative confocal projections from dsLacZ (c) and dsCLIPA2 (d) mosquito midguts dissected $26 \mathrm{~h}$ after infection with $P$. berghei. Live $P$. berghei ookinetes expressing GFP are green (arrows).

data, we expect CLIPA2 to colocalize with TEP1 on surfaces of Plasmodium ookinetes as well as on other microbes coming in contact with the hemolymph. This is supported by the fact that CLIPA2 loss from the hemolymph paralleled that of TEP1 in 2 different experimental settings: bioparticle-injected LacZ kd mosquitoes (fig. 3a) and LRIM1 kd naive mosquitoes (fig. 4). The concomitant loss of CLIPA 2 and TEP $1_{\text {cut }}$ from the hemolymph of LRIM1 kd mosquitoes and the rescue of CLIPA2 loss after cosilencing LRIM1 and TEP1 indicate that CLIPA2 acts downstream of TEP1, and it is an integral component of the mosquito complement-like pathway. CLIPA2 is the second protein after SPCLIP1 shown to be depleted from

Negative Regulation of Mosquito

Complement

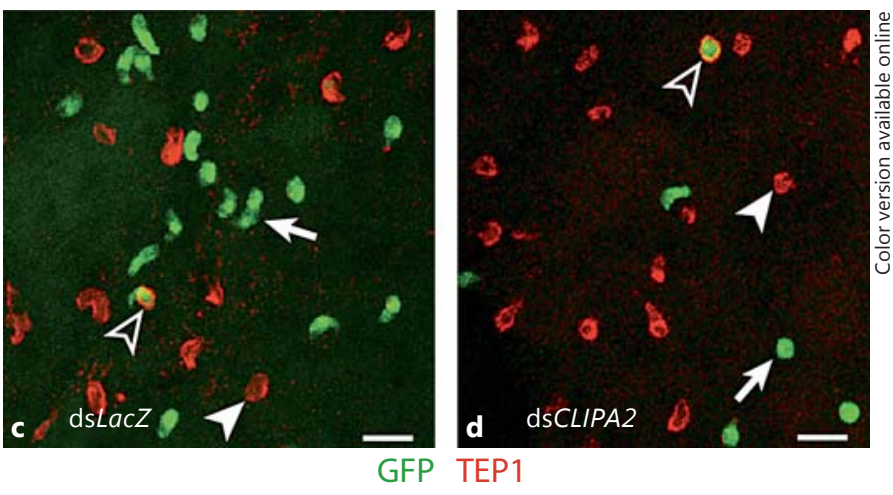

\begin{tabular}{|lclll|}
\hline \multicolumn{2}{l}{ Experiment $1(\mathrm{p}<0.0001)$} \\
\hline Gene kd & GFP+ & TEP+ & GFP+/TEP+ & Melanized \\
\hline LacZ (798) & 54 & 43.9 & 1.8 & 0.4 \\
CLIPA2 (742) & 36.1 & 52.7 & 1.8 & 9.4 \\
Experiment 2 ( $<<0.0001)$ & & & \\
\hline Gene kd & GFP+ & TEP+ & GFP+/TEP+ & Melanized \\
\hline LacZ (433) & 74.4 & 24.2 & 0.5 & 0.9 \\
CLIPA2 (478) & 40.2 & 49.6 & 0.8 & 9.4 \\
\hline
\end{tabular}

TEP1-stained (red) ookinetes are fragmented and have lost the GFP signal (filled arrowheads). There is an increased number of TEP1-stained ookinetes (dead) in midguts of dsCLIPA2 mosquitoes relative to dsLacZ controls. Note the rare presence of ookinetes that are TEP1+ and GFP+ (open arrowheads), and which are most likely in the early death phase. Scale bars are $10 \mu \mathrm{m}$. e Ookinete percentages in the 4 indicated groups [GFP+ (live), TEP1+ (dead), GFP+/TEP1+ (dying) and melanized (dead)] from 2 independent biological experiments. Approximately, 10 midguts were processed per genotype in each experiment. The total number of ookinetes scored per genotype is shown in parentheses. $\mathrm{p}$ values were calculated by the $\chi^{2}$ analysis comparing percentages of TEP1+ ookinetes (these include TEP+, GFP+/TEP+ and melanized) between the 2 mosquito genotypes.

the hemolymph of LRIM1 kd mosquitoes as a consequence of TEP $1_{\text {cut }}$ deposition on tissues, suggesting that this loss of TEP $1_{\text {cut }}$ is not a mere form of protein precipitation as previously suggested [19] but is rather indicative of an autoimmune attack. Unidentified tissue-specific factors likely act to prevent this attack from proceeding to a full-blown response causing immunopathology since TEP1-F levels remain unchanged after silencing LRIM1 (fig. 4) [12]. Additionally, melanization has not been observed in abdomens of LRIM1 kd mosquitoes [Osta M.A., pers. commun.]. The loss of CLIPA2 from the hemolymph of bioparticle-challenged $d s L a c Z$ mosquitoes is replenished later probably through the action of immune 


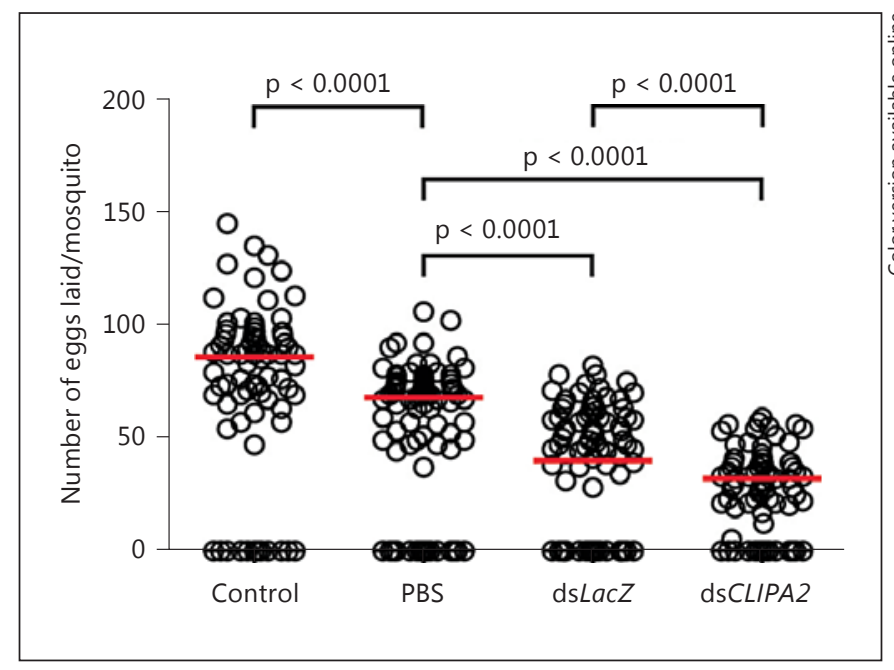

Fig. 6. Infection-induced reduction in fecundity is further enhanced in CLIPA2 $\mathrm{kd}$ mosquitoes. Mosquitoes treated with ds $L a c Z$ and dsCLIPA2 were injected with live E. coli $\left(\mathrm{OD}_{600}=0.4\right)$. Noninjected and PBS-injected wild-type naive mosquitoes served as controls. All experimental groups were fed on the same naive mouse for an equal period of time. The scatter plot represents the median number (red lines) of eggs per mosquito in each experimental group. Statistical analysis was performed using the MannWhitney test. Medians were considered significant if $\mathrm{p}<0.05$.

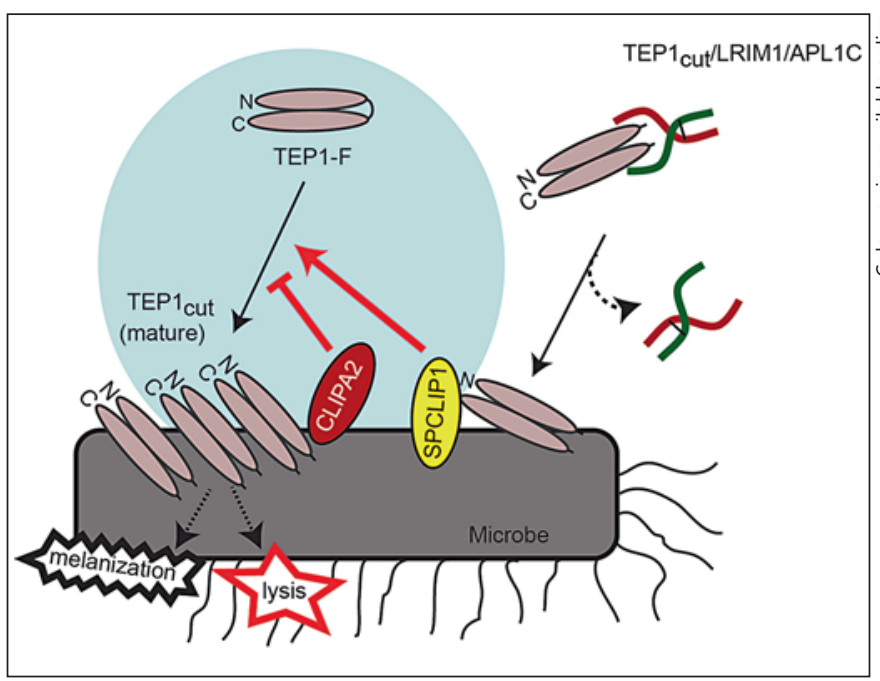

Fig. 7. Model of CLIPA2 function. TEP $1_{\text {cut }}$ released from the LRIM1/APL1C complex (shown in red and green) on microbial surfaces triggers the formation of a putative convertase which requires SPCLIP1 function to process TEP1-F into more TEP $1_{\text {cut }}$. Mature TEP $1_{\text {cut }}$ binds to microbial surfaces initiating several effector reactions including lysis and melanization. CLIPA2 seems to function as a negative regulator of the putative convertase, hence controlling the extent of TEP1-F processing. The N- and C-terminal domains of TEP1 are shown as elliptic structures. The convertase activity is highlighted by a blue circle. signaling pathways controlling the activation of NF- $\kappa \mathrm{B}$, STAT [reviewed in 35] or AP-1 [36] transcription factors, which all have been shown to be involved in mosquito immunity. Which of these pathways contributes most to CLIPA2 replenishment remains to be elucidated.

It is intriguing that the two CLIPs that were shown so far to regulate TEP1-F consumption are noncatalytic, which raises the possibility that they might be regulating the efficiency of TEP1-F cleavage. Le et al. [37] showed that limited activation cleavage of recombinant TEP1-F in vitro resulted in a meta TEP $1_{\text {cut }}$ form which slowly and spontaneously converted to mature TEP $1_{\text {cut }}$ that is captured and stabilized by the LRIM1/APL1C complex. TEP $1_{\text {cut }}$ bound to microbial surfaces may originate from two different sources (fig. 7): the first is the LRIM1/APL1C complex that is thought to deliver its TEP1 $1_{\text {cut }}$ cargo onto microbial surfaces $[19,20,38]$. The second source is a putative TEP1 convertase triggered locally by TEP $1_{\text {cut }}$ originating from the LRIM1/APL1C complex [12]. Once formed this convertase is thought to convert TEP1-F into mature TEP $1_{\text {cut }}$, hence amplifying the response. In this context, our data suggest a role for CLIPA2 in the negative regulation of TEP1 convertase, thus controlling the extent of TEP1-F conversion to TEP $1_{\text {cut }}$ on microbial surfaces. Since CLIPA2 lacks catalytic activity, it is tempting to speculate that it might also act as a molecular chaperon to regulate the conversion of meta TEP $1_{\text {cut }}$ to the mature form in vivo. CLIPA 2 contains $2 \mathrm{~N}$ terminal clip domains suggesting its potential interaction with multiple proteins in the TEP1 pathway. The clip domain has been shown to serve as a module essential for binding cleaved phenoloxidase and the subsequent formation of active phenoloxidase clusters [39, 40]. The future decoding of the CLIPA2 interactome is expected to reveal additional insight into its exact role in the mosquito complement-like pathway.

When survival (i.e. health) was used as a parameter to measure mosquito tolerance to infection, CLIPA2 kd mosquitoes exhibited increased tolerance to systemic E. coli injections and natural B. bassiana infections, compared to controls. Tolerance is a host defense strategy involving a set of mechanisms, most of which are still poorly characterized, that limit the impact of infection on host fitness [41]. A plausible explanation for the increased tolerance of CLIPA2 $\mathrm{kd}$ mosquitoes is the quick clearance or control of microbial growth triggered by the enhanced TEP1-mediated response, possibly limiting infection-induced tissue damage. There is increasing evidence that tissue damage can affect host tolerance to infection [42]. However, when reproductive success was considered as a parameter to measure the tolerance of CLIPA2 kd mosquitoes to systemic E. coli in- 
fections, a reduced tolerance was noted, suggesting the existence of a trade-off between TEP1-mediated immunity and fecundity. In fact, the first molecular link between these two processes was provided by Rono et al. [34] who showed that depletion of the nutrient transport proteins vitellogenin and lipophorin, required for oogenesis, enhanced TEP1-mediated killing of $P$. berghei ookinetes in midguts of A. gambiae mosquitoes [34]. It remains to be determined how the enhanced TEP1-mediated response in CLIPA2 kd mosquitoes reduced fecundity and whether it entails redistribution of energy resources or altering the expression of nutrient transport proteins.

Another interesting result that emerged from our study is the functional link between mosquito complement and PPO activation. We recently showed that hemolymph PPO activation following systemic bacterial injections as well as the activation cleavage of CLIPA8, a key regulator of the mosquito melanization response [18, 21, 27], require the functions of both, TEP1 and SPCLIP1, suggesting the existence of a functional link between TEP1 and PPO activation [12]. Here, we provide further evidence in support of this link by showing that the enhanced TEP1mediated response in CLIPA2 $\mathrm{kd}$ mosquitoes was paralleled by an abnormal increase in hemolymph phenoloxidase activity. Based on these observations, we propose that in A. gambiae the infection-induced melanization re- sponse may constitute an effector arm of the complementlike pathway. It remains to be elucidated how TEP1 triggers PPO activation at the molecular level and why Plasmodium ookinetes rarely become melanized in susceptible mosquitoes despite being bound by TEP1, while bacteria $[12,27]$ and fungi $[18]$ are efficiently melanized.

In conclusion, there is increasing evidence that TEP1 consumption during systemic immune responses is subject to tight regulation possibly to establish a balance between an efficient immune response on one hand and minimal fitness costs on the other. Identifying the components of this regulatory network is essential if we are to build a comprehensive model of the activation and regulation of this ancient complement-like system and define to which extent common strategies exist with that of mammals.

\section{Acknowledgments}

We thank the American University of Beirut (AUB) Imaging Core Facility for granting free access to the confocal microscope and Kamal A. Shair Central Research Science Laboratory at AUB for providing free access to its equipment. This work was funded by the AUB University Research Board and the Lebanese National Council for Scientific Research (to M.A.O.) and Wellcome Trust project (093587/Z/10/Z; to G.K.C.). L.K. was supported by a fellowship from the Lebanese National Council for Scientific Research.

\section{References}

1 Lemaitre B, Hoffmann J: The host defense of Drosophila melanogaster. Annu Rev Immunol 2007;25:697-743.

2 Kurata S, Ariki S, Kawabata S: Recognition of pathogens and activation of immune responses in drosophila and horseshoe crab innate immunity. Immunobiology 2006;211:237-249.

$\checkmark 3$ Cerenius L, Lee BL, Soderhall K: The proPOsystem: pros and cons for its role in invertebrate immunity. Trends Immunol 2008;29:263-271.

-4 Lee SY, Kwon TH, Hyun JH, Choi JS, Kawabata SI, Iwanaga S, Lee BL: In vitro activation of pro-phenol-oxidase by two kinds of pro-phenol-oxidase-activating factors isolated from hemolymph of coleopteran, Holotrichia diomphalia larvae. Eur J Biochem 1998;254:50-57.

5 Kwon TH, Kim MS, Choi HW, Joo CH, Cho MY, Lee BL: A masquerade-like serine proteinase homologue is necessary for phenoloxidase activity in the coleopteran insect, Holotrichia diomphalia larvae. Eur J Biochem 2000;267:6188-6196.

6 Lee KY, Zhang R, Kim MS, Park JW, Park HY, Kawabata S, Lee BL: A zymogen form of masquerade-like serine proteinase homologue is cleaved during pro-phenoloxidase activation by $\mathrm{Ca}^{2+}$ in coleopteran and Tene- brio molitor larvae. Eur J Biochem 2002;269: 4375-4383.

7 Yu XQ, Jiang H, Wang Y, Kanost MR: Nonproteolytic serine proteinase homologs are involved in prophenoloxidase activation in the tobacco hornworm, Manduca sexta. Insect Biochem Mol Biol 2003;33:197-208.

$\checkmark 8$ Murugasu-Oei B, Rodrigues V, Yang X, Chia W: Masquerade: a novel secreted serine protease-like molecule is required for somatic muscle attachment in the Drosophila embryo. Genes Dev 1995;9:139-154.

-9 Lee SY, Soderhall K: Characterization of a pattern recognition protein, a masquerade-like protein, in the freshwater crayfish Pacifastacus leniusculus. J Immunol 2001;166:7319-7326.

10 Bartholomay LC, Waterhouse RM, Mayhew GF, Campbell CL, Michel K, Zou Z, Ramirez JL, Das S, Alvarez K, Arensburger P, Bryant B, Chapman SB, Dong Y, Erickson SM, Karunaratne SH, Kokoza V, Kodira CD, Pignatelli $\mathrm{P}$, Shin SW, Vanlandingham DL, Atkinson PW, Birren B, Christophides GK, Clem RJ, Hemingway J, Higgs S, Megy K, Ranson H, Zdobnov EM, Raikhel AS, Christensen BM, Dimopoulos G, Muskavitch MA: Pathogenomics of Culex quinquefasciatus and meta-analysis of infection responses to diverse pathogens. Science 2010;330:88-90.

11 Waterhouse RM, Kriventseva EV, Meister S, Xi Z, Alvarez KS, Bartholomay LC, BarillasMury C, Bian G, Blandin S, Christensen BM, Dong Y, Jiang H, Kanost MR, Koutsos AC, Levashina EA, Li J, Ligoxygakis P, Maccallum RM, Mayhew GF, Mendes A, Michel K, Osta MA, Paskewitz S, Shin SW, Vlachou D, Wang L, Wei W, Zheng L, Zou Z, Severson DW, Raikhel AS, Kafatos FC, Dimopoulos G, Zdobnov EM, Christophides GK: Evolutionary dynamics of immune-related genes and pathways in disease-vector mosquitoes. Science 2007;316:1738-1743

12 Povelones M, Bhagavatula L, Yassine H, Tan LA, Upton LM, Osta MA, Christophides GK: The CLIP-domain serine protease homolog SPCLIP1 regulates complement recruitment to microbial surfaces in the malaria mosquito Anopheles gambiae. PLoS Pathog 2013; 9:e1003623.

13 Blandin S, Shiao SH, Moita LF, Janse CJ, Waters AP, Kafatos FC, Levashina EA: Complement-like protein TEP1 is a determinant of vectorial capacity in the malaria vector Anopheles gambiae. Cell 2004;116:661-670. 
14 Dong Y, Aguilar R, Xi Z, Warr E, Mongin E, Dimopoulos G: Anopheles gambiae immune responses to human and rodent Plasmodium parasite species. PLoS Pathog 2006; 2:e52.

15 Molina-Cruz A, DeJong RJ, Ortega C, Haile A, Abban E, Rodrigues J, Jaramillo-Gutierrez G, Barillas-Mury C: Some strains of Plasmodium falciparum, a human malaria parasite, evade the complement-like system of Anopheles gambiae mosquitoes. Proc Natl Acad Sci USA 2012;109:E1957-E1962.

16 Levashina EA, Moita LF, Blandin S, Vriend G, Lagueux M, Kafatos FC: Conserved role of a complement-like protein in phagocytosis revealed by dsRNA knockout in cultured cells of the mosquito, Anopheles gambiae. Cell 2001;104:709-718.

17 Moita LF, Wang-Sattler R, Michel K, Zimmermann T, Blandin S, Levashina EA, Kafatos FC: In vivo identification of novel regulators and conserved pathways of phagocytosis in A. gambiae. Immunity 2005;23. 65-73.

18 Yassine H, Kamareddine L, Osta MA: The mosquito melanization response is implicated in defense against the entomopathogenic fungus Beauveria bassiana. PLoS Pathog 2012;8:e1003029.

19 Fraiture M, Baxter RH, Steinert S, Chelliah Y, Frolet C, Quispe-Tintaya W, Hoffmann JA, Blandin SA, Levashina EA: Two mosquito LRR proteins function as complement control factors in the TEP1-mediated killing of Plasmodium. Cell Host Microbe 2009;5:273284.

20 Povelones M, Waterhouse RM, Kafatos FC, Christophides GK: Leucine-rich repeat protein complex activates mosquito complement in defense against Plasmodium parasites. Science 2009;324:258-261.

21 Volz J, Muller HM, Zdanowicz A, Kafatos FC, Osta MA: A genetic module regulates the melanization response of Anopheles to Plasmodium. Cell Microbiol 2006;8:1392-1405.
2 Franke-Fayard B, Trueman H, Ramesar J, Mendoza J, van der Keur M, van der Linden R, Sinden RE, Waters AP, Janse CJ: A Plasmodium berghei reference line that constitutively expresses GFP at a high level throughout the complete life cycle. Mol Biochem Parasitol 2004;137:23-33.

23 Blandin S, Moita LF, Kocher T, Wilm M, Kafatos FC, Levashina EA: Reverse genetics in the mosquito Anopheles gambiae: targeted disruption of the defensin gene. EMBO Rep 2002;3:852-856.

24 Schnitger AK, Yassine H, Kafatos FC, Osta MA: Two C-type lectins cooperate to defend Anopheles gambiae against Gram-negative bacteria. J Biol Chem 2009;284:17616-17624.

25 Povelones M, Upton LM, Sala KA, Christophides GK: Structure-function analysis of the Anopheles gambiae LRIM1/APL1C complex and its interaction with complement C3-like protein TEP1. PLoS Pathog 2011;7:e1002023.

26 Muller HM, Dimopoulos G, Blass C, Kafatos FC: A hemocyte-like cell line established from the malaria vector Anopheles gambiae expresses six prophenoloxidase genes. J Biol Chem 1999;274:11727-11735.

-27 Schnitger AK, Kafatos FC, Osta MA: The melanization reaction is not required for survival of Anopheles gambiae mosquitoes after bacterial infections. J Biol Chem 2007;282:21884-21888.

28 Povelones ML, Tiengwe C, Gluenz E, Gull K, Englund PT, Jensen RE: Mitochondrial shape and function in trypanosomes requires the outer membrane protein, TBLOK1. Mol Microbiol 2013;87:713-729.

29 Tabak LA: The role of mucin-type O-glycans in eukaryotic development. Semin Cell Dev Biol 2010;21:616-621.

30 Daley WP, Peters SB, Larsen M: Extracellular matrix dynamics in development and regenerative medicine. J Cell Sci 2008;121:255-264.

31 Dharmani P, Srivastava V, Kissoon-Singh V, Chadee K: Role of intestinal mucins in innate host defense mechanisms against pathogens. J Innate Immun 2009;1:123-135.

-32 King JG, Hillyer JF: Infection-induced interaction between the mosquito circulatory and immune systems. PLoS Pathog 2012;8:e1003058.
33 Ayres JS, Schneider DS: Tolerance of infections. Annu Rev Immunol 2012;30:271-294.

34 Rono MK, Whitten MM, Oulad-Abdelghani $\mathrm{M}$, Levashina EA, Marois E: The major yolk protein vitellogenin interferes with the antiplasmodium response in the malaria mosquito Anopheles gambiae. PLoS Biol 2010; 8:e1000434

35 Clayton AM, Dong Y, Dimopoulos G: The Anopheles innate immune system in the defense against malaria infection. J Innate Immun 2014;6:169-181.

36 Garver LS, de Almeida Oliveira G, BarillasMury C: The JNK pathway is a key mediator of Anopheles gambiae antiplasmodial immunity. PLoS Pathog 2013;9:e1003622.

-37 Le BV, Williams M, Logarajah S, Baxter RH Molecular basis for genetic resistance of Anopheles gambiae to Plasmodium: structural analysis of TEP1 susceptible and resistant alleles. PLoS Pathog 2012;8:e1002958.

- 38 Baxter RH, Steinert S, Chelliah Y, Volohonsky G, Levashina EA, Deisenhofer J: A heterodimeric complex of the LRR proteins LRIM1 and APL1C regulates complementlike immunity in Anopheles gambiae. Proc Natl Acad Sci USA 2010;107:16817-16822.

39 Huang R, Lu Z, Dai H, Velde DV, Prakash O, Jiang $\mathrm{H}$ : The solution structure of clip domains from Manduca sexta prophenoloxidase activating proteinase-2. Biochemistry 2007;46:11431-11439.

40 Piao S, Song YL, Kim JH, Park SY, Park JW, Lee BL, Oh BH, Ha NC: Crystal structure of a clip-domain serine protease and functional roles of the clip domains. EMBO J 2005;24: 4404-4414.

41 Medzhitov R, Schneider DS, Soares MP: Disease tolerance as a defense strategy. Science 2012;335:936-941.

42 Jamieson AM, Pasman L, Yu S, Gamradt P, Homer RJ, Decker T, Medzhitov R: Role of tissue protection in lethal respiratory viralbacterial coinfection. Science 2013;340:1230 1234. 